\title{
LiangPeng Xiong
}

\section{SOME GENERAL RESULTS AND EXTREME POINTS OF p-VALENT FUNCTIONS WITH NEGATIVE COEFFICIENTS}

\begin{abstract}
In this paper, we further investigate the class of functions $\phi^{*}(n, p, \lambda, \alpha)$ which are analytic in the open unit disk $\triangle=\{z:|z|<1\}$, and involve the combinations of the representations of p-valently starlike and convex functions. We obtain several generalized results on the modified-Hadamard product of the class $\phi^{*}(n, p, \lambda, \alpha)$, which extend the corresponding results obtained by Altintaş et al. [Computers Math. Applic. 30(2), 9-16, (1995)]; Darwish, Aouf [Mathematical and Computer Modelling(2007), doi:10.1016/j.mcm.2007.08.016]. Futher, by fixing the second coefficient of functions in $\phi^{*}(n, p, \lambda, \alpha)$, we introduce the special class $\phi_{c}^{*}(n, p, \lambda, \alpha)$ and discuss the extreme points.
\end{abstract}

\section{Introduction}

Let $S(n, p)$ denote the class of functions of the form

$$
f(z)=z^{p}-\sum_{k=n+p}^{\infty} a_{k} z^{k} \quad\left(a_{k} \geq 0, n, p \in N=\{1,2, \ldots\}\right)
$$

which are analytic and p-valent in the unit disk $\triangle=\{z:|z|<1\}$.

We denote the class of p-valent starlike functions of order $\alpha$ and the p-valent convex functions of order $\alpha$ by $T_{n}(p, \alpha), C_{n}(p, \alpha), 0 \leq \alpha<p$, respectively, where

$$
T_{n}(p, \alpha)=\left\{f(z): \operatorname{Re} \frac{z f^{\prime}(z)}{f(z)}>\alpha, f(z) \in S(n, p), z \in \triangle\right\}
$$

and

$$
C_{n}(p, \alpha)=\left\{f(z): \operatorname{Re}\left(1+\frac{z f^{\prime \prime}(z)}{f^{\prime}(z)}\right)>\alpha, f(z) \in S(n, p), z \in \triangle\right\} .
$$

2000 Mathematics Subject Classification: 30C45.

Key words and phrases: analytic functions, modified-Hadamard product, extreme points. 
Owa [1] have studied the classes $T_{n}(p, \alpha)$ and $C_{n}(p, \alpha)$, and H. E. Darwish, M. K. Aouf [2] further investigate them. Special cases of them have been considered by Srivastava et al. [3] and Domokos [4] with $T_{n}(1, \alpha), C_{n}(1, \alpha)$.

A function $f(z) \in S(n, p)$ is said to be in the class $\phi^{*}(n, p, \lambda, \alpha)$ if and only if

$$
\operatorname{Re}\left\{\frac{z f^{\prime}(z)+\lambda z^{2} f^{\prime \prime}(z)}{\lambda z f^{\prime}(z)+(1-\lambda) f(z)}\right\}>\alpha \quad(0 \leq \alpha<p, 0 \leq \lambda \leq 1, z \in \triangle) .
$$

The class $\phi^{*}(n, p, \lambda, \alpha)$ was studied by Altintaş et al. [5] and Altintaş et al. [6]. In particular, $\phi^{*}(n, 1, \lambda, \alpha) \equiv p(n, \lambda, \alpha)$ was considered earlier by Altintaş [7]. We can note that $\phi^{*}(n, p, 0, \alpha) \equiv T_{n}(p, \alpha), \phi^{*}(n, p, 1, \alpha) \equiv$ $C_{n}(p, \alpha)$, therefore, the class $\phi^{*}(n, p, \lambda, \alpha)$ is the generalization of $C_{n}(p, \alpha)$ and $T_{n}(p, \alpha)$.

Now we can fix the second coefficient of functions in $\phi^{*}(n, p, \lambda, \alpha)$ and obtain the following class:

$$
\begin{gathered}
\phi_{c}^{*}(n, p, \lambda, \alpha)=\left\{f(z): f(z)=z^{p}-a_{n+p} z^{n+p}-\sum_{k=n+p+1}^{\infty} a_{k} z^{k} \in \phi^{*}(n, p, \lambda, \alpha),\right. \\
\left.a_{n+p}=\frac{c(p-\alpha)[\lambda(p-1)+1]}{(n+p-\alpha)[\lambda(n+p-1)+1]}, 0 \leq c \leq 1\right\} .
\end{gathered}
$$

In the present paper, we will obtain several generalized results on $C_{n}(p, \alpha)$ and $T_{n}(p, \alpha)$ by further investigating $\phi^{*}(n, p, \lambda, \alpha)$, which mainly extend the corresponding results obtained by Altintaş et al. [5], Owa [1], H. E. Darwish, M. K. Aouf [2], Domokos [4]. Also we discuss the extreme points of the $\phi_{c}^{*}(n, p, \lambda, \alpha)$.

\section{The general results on two modified-Hadamard products}

In order to investigate the class $\phi^{*}(n, p, \lambda, \alpha)$, we begin with the following Lemma given by Altintaş et al. [5]:

Lemma 1. If the function $f(z) \in S(n, p)$, then $f(z)$ is in the class $\phi^{*}(n, p, \lambda, \alpha)$ if and only if

$$
\sum_{k=n+p}^{\infty}(k-\alpha)[\lambda(k-1)+1] a_{k} \leq(p-\alpha)[\lambda(p-1)+1] \quad(n, p \in N) .
$$

Let

$$
f_{j}(z)=z^{p}-\sum_{k=n+p}^{\infty} a_{k, j} z^{k} \quad\left(a_{k, j} \geq 0 ; j=1,2, \ldots, m ; p, n \in N\right) .
$$


We define the generalized modified-Hadamard product $f_{1} * f_{2} *, \ldots$, $* f_{m}(z)$ by

$$
f_{1} * f_{2} *, \ldots, * f_{m}(z)=z^{p}-\sum_{k=n+p}^{\infty} a_{k, 1} a_{k, 2} \ldots a_{k, m} z^{k}
$$

Theorem 1. If the functions $f_{j}(z)=z^{p}-\sum_{k=n+p}^{\infty} a_{k, j} z^{k} \in \phi^{*}\left(n, p, \lambda, \alpha_{j}\right)$, $j=1,2 \ldots, m$, then $f_{1} * f_{2} * \cdots * f_{m}(z) \in \phi^{*}\left(n, p, \lambda, \gamma_{m}\right)$ when $n \geq p$, where $\gamma_{m}$ is determined by

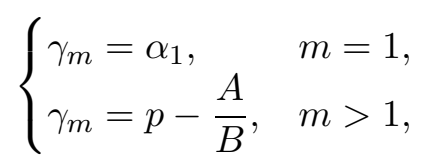

where $A=n(\lambda p-\lambda+1)\left(p-\gamma_{m-1}\right)\left(p-\alpha_{m}\right), B=[\lambda(n+p)-\lambda+1](n+p-$ $\left.\gamma_{m-1}\right)\left(n+p-\alpha_{m}\right)-(\lambda p-\lambda+1)\left(p-\gamma_{m-1}\right)\left(p-\alpha_{m}\right)$.

The result is sharp for the functions $f(z)$ given by

$$
f(z)=z^{p}-\frac{\left(p-\alpha_{j}\right)[\lambda(p-1)+1]}{\left(n+p-\alpha_{j}\right)[\lambda(n+p-1)+1]} z^{n+p}, j=1,2, \ldots, m ; n, p \in N .
$$

Proof. First we need to prove the theorem when $m=2$.

Suppose the functions

$$
f_{j}(z)=z^{p}-\sum_{k=n+p}^{\infty} a_{k, j} z^{k} \in \phi^{*}\left(n, p, \lambda, \alpha_{j}\right), j=1,2,
$$

then from Lemma 1, we have

$$
\sum_{k=n+p}^{\infty} \frac{\left(k-\alpha_{j}\right)[\lambda(k-1)+1]}{\left(p-\alpha_{j}\right)[\lambda(p-1)+1]} a_{k, j} \leq 1, j=1,2,
$$

moreover

$$
\left\{\sum_{k=n+p}^{\infty} \frac{\left(k-\alpha_{j}\right)[\lambda(k-1)+1]}{\left(p-\alpha_{j}\right)[\lambda(p-1)+1]} a_{k, j}\right\}^{\frac{1}{2}} \leq 1, j=1,2 .
$$

By using the Holder inequality, we get

$$
\sum_{k=n+p}^{\infty}\left[\frac{\left(k-\alpha_{1}\right)(\lambda k-\lambda+1)}{\left(p-\alpha_{1}\right)(\lambda p-\lambda+1)}\right]^{\frac{1}{2}}\left[\frac{\left(k-\alpha_{2}\right)(\lambda k-\lambda+1)}{\left(p-\alpha_{2}\right)(\lambda p-\lambda+1)}\right]^{\frac{1}{2}} a_{k, 1}^{\frac{1}{2}} a_{k, 2}^{\frac{1}{2}} \leq 1 .
$$

So

$$
\sum_{k=n+p}^{\infty} \frac{\lambda k-\lambda+1}{\lambda p-\lambda+1}\left(\frac{k-\alpha_{1}}{p-\alpha_{1}}\right)^{\frac{1}{2}}\left(\frac{k-\alpha_{2}}{p-\alpha_{2}}\right)^{\frac{1}{2}} \sqrt{a_{k, 1} a_{k, 2}} \leq 1
$$


and

$$
\frac{1}{\sqrt{a_{k, 1} a_{k, 2}}} \geq \frac{\lambda k-\lambda+1}{\lambda p-\lambda+1}\left(\frac{k-\alpha_{1}}{p-\alpha_{1}}\right)^{\frac{1}{2}}\left(\frac{k-\alpha_{2}}{p-\alpha_{2}}\right)^{\frac{1}{2}} \quad(k \geq n+p) .
$$

We have to find the largest $\gamma_{2}$ such that

$$
\sum_{k=n+p}^{\infty} \frac{\left(k-\gamma_{2}\right)[\lambda k-\lambda+1]}{\left(p-\gamma_{2}\right)[\lambda p-\lambda+1]} a_{k, 1} a_{k, 2} \leq 1
$$

From (2.3), (2.5) hold true if

$$
\frac{k-\gamma_{2}}{p-\gamma_{2}} a_{k, 1} a_{k, 2} \leq\left(\frac{k-\alpha_{1}}{p-\alpha_{1}}\right)^{\frac{1}{2}}\left(\frac{k-\alpha_{2}}{p-\alpha_{2}}\right)^{\frac{1}{2}} \sqrt{a_{k, 1} a_{k, 2}} \quad(k \geq n+p)
$$

or

$$
\frac{k-\gamma_{2}}{p-\gamma_{2}} \leq\left(\frac{k-\alpha_{1}}{p-\alpha_{1}}\right)^{\frac{1}{2}}\left(\frac{k-\alpha_{2}}{p-\alpha_{2}}\right)^{\frac{1}{2}} \frac{1}{\sqrt{a_{k, 1} a_{k, 2}}} \quad(k \geq n+p) .
$$

From (2.4), (2.6) is equal to

$$
\begin{array}{r}
\gamma_{2} \leq p-\frac{(k-p)(\lambda p-\lambda+1)\left(p-\alpha_{1}\right)\left(p-\alpha_{2}\right)}{(\lambda k-\lambda+1)\left(k-\alpha_{1}\right)\left(k-\alpha_{2}\right)-(\lambda p-\lambda+1)\left(p-\alpha_{1}\right)\left(p-\alpha_{2}\right)} \\
(k \geq n+p) .
\end{array}
$$

Now let

$$
g(k)=p-\frac{(k-p)(\lambda p-\lambda+1)\left(p-\alpha_{1}\right)\left(p-\alpha_{2}\right)}{(\lambda k-\lambda+1)\left(k-\alpha_{1}\right)\left(k-\alpha_{2}\right)-(\lambda p-\lambda+1)\left(p-\alpha_{1}\right)\left(p-\alpha_{2}\right)},
$$

then $g^{\prime}(k)=\frac{A(k)}{B(k)}$, where

$$
\begin{aligned}
B(k)= & {\left[(\lambda k-\lambda+1)\left(k-\alpha_{1}\right)\left(k-\alpha_{2}\right)-(\lambda p-\lambda+1)\left(p-\alpha_{1}\right)\left(p-\alpha_{2}\right)\right]^{2}>0, } \\
A(k)= & \lambda(k-p)(\lambda p-\lambda+1)\left(p-\alpha_{1}\right)\left(p-\alpha_{2}\right)\left(k-\alpha_{1}\right)\left(k-\alpha_{2}\right) \\
& +(\lambda p-\lambda+1)^{2}\left(p-\alpha_{1}\right)^{2}\left(p-\alpha_{2}\right)^{2} \\
& +(\lambda p-\lambda+1)(\lambda k-\lambda+1)\left(p-\alpha_{1}\right)\left(p-\alpha_{2}\right)\left[k(k-2 p)+\alpha_{1}\left(p-\alpha_{2}\right)+p \alpha_{2}\right] .
\end{aligned}
$$

Since $n \geq p$, we have $k \geq n+p \geq 2 p$, so $A(k) \geq 0$, it implies that $g(k)$ is increasing in $\mathrm{k}$. This gives that

$$
\begin{aligned}
& \gamma_{2} \leq g(n+p) \\
= & p-\frac{n(\lambda p-\lambda+1)\left(p-\alpha_{1}\right)\left(p-\alpha_{2}\right)}{[\lambda(n+p)-\lambda+1]\left(n+p-\alpha_{1}\right)\left(n+p-\alpha_{2}\right)-(\lambda p-\lambda+1)\left(p-\alpha_{1}\right)\left(p-\alpha_{2}\right)} .
\end{aligned}
$$

Therefore the result is true for $m=2$. 
When $m=3$,

$$
f_{1} * f_{2} * f_{3}(z)=z^{p}-\sum_{k=n+p}^{\infty} a_{k, 1} a_{k, 2} a_{k, 3} z^{k}=z^{p}-\sum_{k=n+p}^{\infty} \omega_{k} a_{k, 3} z^{k},
$$

where $\omega_{k}=a_{k, 1} a_{k, 2}$.

From (2.7), we can know $f_{1} * f_{2}(z)=z^{p}-\sum_{k=n+p}^{\infty} w_{k} z^{k} \in \phi^{*}\left(n, p, \lambda, \gamma_{2}\right)$, use the $\omega_{k}$ and $a_{k, 3}$ take place of the $a_{k, 1}$ and $a_{k, 2}$ of (2.2), respectively, therefore we have changed the case $m=3$ to the case $m=2$, it is easy to obtain

$$
\gamma_{3} \leq p-\frac{n(\lambda p-\lambda+1)\left(p-\gamma_{2}\right)\left(p-\alpha_{3}\right)}{[\lambda(n+p)-\lambda+1]\left(n+p-\gamma_{2}\right)\left(n+p-\alpha_{3}\right)-(\lambda p-\lambda+1)\left(p-\gamma_{2}\right)\left(p-\alpha_{3}\right)} .
$$

Take the same process, we can prove the $\gamma_{4}, \gamma_{5}, \ldots, \gamma_{m}$. So the

$\gamma_{m} \leq$

$$
p-\frac{n(\lambda p-\lambda+1)\left(p-\gamma_{m-1}\right)\left(p-\alpha_{m}\right)}{[\lambda(n+p)-\lambda+1]\left(n+p-\gamma_{m-1}\right)\left(n+p-\alpha_{m}\right)-(\lambda p-\lambda+1)\left(p-\gamma_{m-1}\right)\left(p-\alpha_{m}\right)}
$$

is true.

A special case of Theorem 1 when $\lambda=0$ and $\lambda=1$ yield

Corollary 1. If the functions $f_{j}(z)=z^{p}-\sum_{k=n+p}^{\infty} a_{k, j} z^{k} \in T_{n}\left(p, \alpha_{j}\right)$, $j=1,2 \ldots, m$, then $f_{1} * f_{2} * \cdots * f_{m}(z) \in T_{n}\left(p, \gamma_{m}\right)$ when $n \geq p$, where $\gamma_{m}$ is determined by

$$
\begin{cases}\gamma_{1}=\alpha_{1}, & m=1, \\ \gamma_{m}=p-\frac{n\left(p-\gamma_{m-1}\right)\left(p-\alpha_{m}\right)}{\left(n+p-\gamma_{m-1}\right)\left(n+p-\alpha_{m}\right)-\left(p-\gamma_{m-1}\right)\left(p-\alpha_{m}\right)}, & m>1 .\end{cases}
$$

The result is sharp for the functions $f(z)$ given by

$$
f(z)=z^{p}-\frac{p-\alpha_{j}}{n+p-\alpha_{j}} z^{n+p}, \quad j=1,2, \ldots, m ; n, p \in N .
$$

Corollary 2. If the functions $f_{j}(z)=z^{p}-\sum_{k=n+p}^{\infty} a_{k, j} z^{k} \in C_{n}\left(p, \alpha_{j}\right)$, $4 j=1,2 \ldots, m$, then $f_{1} * f_{2} * \cdots * f_{m}(z) \in C_{n}\left(p, \gamma_{m}\right)$ when $n \geq p$, where $\gamma_{m}$ is determined by

$$
\left\{\begin{array}{lr}
\gamma_{1}=\alpha_{1}, & m=1, \\
\gamma_{m}=p-\frac{n p\left(p-\gamma_{m-1}\right)\left(p-\alpha_{m}\right)}{(n+p)\left(n+p-\gamma_{m-1}\right)\left(n+p-\alpha_{m}\right)-p\left(p-\gamma_{m-1}\right)\left(p-\alpha_{m}\right)}, \\
m>1 .
\end{array}\right.
$$


The result is sharp for the functions $f(z)$ given by

$$
f(z)=z^{p}-\frac{p\left(p-\alpha_{j}\right)}{(n+p)\left(n+p-\alpha_{j}\right)} z^{n+p}, \quad j=1,2, \ldots, m ; n, p \in N .
$$

REMARK 1. Taking $m=2, \alpha_{1}=\alpha_{2}=\alpha$ in the Theorem 1, we can obtain the result obtained by O. Altintaş et al. [5, Theorem 3.4].

TheOREM 2. If the functions $f_{j}(z)=z^{p}-\sum_{k=n+p}^{\infty} a_{k, j} z^{k} \in \phi^{*}\left(n, p, \lambda, \alpha_{j}\right)$, $j=1,2, \ldots, m$. Define $F_{m}(z)=z^{p}-\sum_{k=n+p}^{\infty}\left(\sum_{j=1}^{m} a_{k, j}^{r}\right) z^{k} \quad(z \in \triangle ; n, p \in$ $N ; r>1)$, then when

$$
p(n+p-\alpha)^{r}[\lambda(n+p-1)+1]^{r-1} \geq m(n+p)(p-\alpha)^{r}[\lambda(p-1)+1]^{r-1},
$$

we have $F_{m}(z) \in \phi^{*}\left(n, p, \lambda, \gamma_{m}\right)$, where

$$
\begin{gathered}
\gamma_{m}=p-\frac{m n(p-\alpha)^{r}[\lambda(p-1)+1]^{r-1}}{(n+p-\alpha)^{r}[\lambda(n+p-1)+1]^{r-1}-m(p-\alpha)^{r}[\lambda(p-1)+1]^{r-1}}, \\
\alpha=\min _{1 \leq j \leq m}\left\{\alpha_{j}\right\} .
\end{gathered}
$$

The result is sharp for the functions $f(z)$ given by

$$
f(z)=z^{p}-\frac{\left(p-\alpha_{j}\right)[\lambda(p-1)+1]}{\left(n+p-\alpha_{j}\right)[\lambda(n+p-1)+1]} z^{n+p}, j=1,2, \ldots, m ; n, p \in N .
$$

Proof. Suppose the functions $f_{j}(z)=z^{p}-\sum_{k=n+p}^{\infty} a_{k, j} z^{k} \in \phi^{*}\left(n, p, \lambda, \alpha_{j}\right), j=$ $1,2, . ., m$, then from Lemma 1 , we have

$$
\sum_{k=n+p}^{\infty} \frac{\left(k-\alpha_{j}\right)[\lambda(k-1)+1]}{\left(p-\alpha_{j}\right)[\lambda(p-1)+1]} a_{k, j} \leq 1, j=1,2 \ldots, m .
$$

By virtue of the Cauchy-Schwarz inequality, we obtain

$$
\begin{aligned}
\sum_{k=n+p}^{\infty}\left\{\frac{\left(k-\alpha_{j}\right)[\lambda(k-1)+1]}{\left(p-\alpha_{j}\right)[\lambda(p-1)+1]}\right\}^{r} a_{k, j}^{r} \\
\leq\left\{\sum_{k=n+p}^{\infty} \frac{\left(k-\alpha_{j}\right)[\lambda(k-1)+1]}{\left(p-\alpha_{j}\right)[\lambda(p-1)+1]} a_{k, j}\right\}^{r} \leq 1, \quad j=1,2, \ldots, m .
\end{aligned}
$$

So

$$
\sum_{k=n+p}^{\infty}\left\{\frac{1}{m} \sum_{j=1}^{m}\left\{\frac{\left(k-\alpha_{j}\right)[\lambda(k-1)+1]}{\left(p-\alpha_{j}\right)[\lambda(p-1)+1]}\right\}^{r} a_{k, j}^{r}\right\} \leq 1
$$


We must find the largest $\gamma_{m}$ such that

$$
\sum_{k=n+p}^{\infty} \frac{\left(k-\gamma_{m}\right)[\lambda(k-1)+1]}{\left(p-\gamma_{m}\right)[\lambda(p-1)+1]}\left(\sum_{j=1}^{m} a_{k, j}^{r}\right) \leq 1,
$$

From (2.9), (2.10) hold true if

$$
\begin{aligned}
\frac{\left(k-\gamma_{m}\right)[\lambda(k-1)+1]}{\left(p-\gamma_{m}\right)[\lambda(p-1)+1]} & \left(\sum_{j=1}^{m} a_{k, j}^{r}\right) \\
\leq & \frac{1}{m} \sum_{j=1}^{m}\left\{\frac{\left(k-\alpha_{j}\right)[\lambda(k-1)+1]}{\left(p-\alpha_{j}\right)[\lambda(p-1)+1]}\right\}^{r} a_{k, j}^{r} \quad(k \geq n+p) .
\end{aligned}
$$

Let

$$
\alpha=\min _{1 \leq j \leq m}\left\{\alpha_{j}\right\}
$$

we have

$$
\begin{aligned}
\frac{1}{m} \sum_{j=1}^{m}\left\{\frac{\left(k-\alpha_{j}\right)[\lambda(k-1)+1]}{\left(p-\alpha_{j}\right)[\lambda(p-1)+1]}\right\}^{r} a_{k, j}^{r} & \geq \frac{1}{m} \sum_{j=1}^{m}\left\{\frac{(k-\alpha)[\lambda(k-1)+1]}{(p-\alpha)[\lambda(p-1)+1]}\right\}^{r} a_{k, j}^{r} \\
& =\frac{1}{m}\left\{\frac{(k-\alpha)[\lambda(k-1)+1]}{(p-\alpha)[\lambda(p-1)+1]}\right\}^{r} \sum_{j=1}^{m} a_{k, j}^{r} .
\end{aligned}
$$

So (2.10) is equivalent to

$$
\frac{\left(k-\gamma_{m}\right)[\lambda(k-1)+1]}{\left(p-\gamma_{m}\right)[\lambda(p-1)+1]} \leq \frac{1}{m}\left\{\frac{(k-\alpha)[\lambda(k-1)+1]}{(p-\alpha)[\lambda(p-1)+1]}\right\}^{r}(k \geq n+p) .
$$

$\mathrm{U}$ sing (2.8), we can know that (2.11) is true if

$$
\begin{aligned}
& \gamma_{m} \leq p-\frac{m(k-p)(p-\alpha)^{r}[\lambda(p-1)+1]^{r-1}}{(k-\alpha)^{r}[\lambda(k-1)+1]^{r-1}-m(p-\alpha)^{r}[\lambda(p-1)+1]^{r-1}} \\
&(k \geq n+p) .
\end{aligned}
$$

Now let

$$
g(k)=p-\frac{m(k-p)(p-\alpha)^{r}[\lambda(p-1)+1]^{r-1}}{(k-\alpha)^{r}[\lambda(k-1)+1]^{r-1}-m(p-\alpha)^{r}[\lambda(p-1)+1]^{r-1}},
$$

then $g^{\prime}(k)=\frac{C(k)}{D(k)}$, where

$$
\begin{aligned}
D(k)= & p(k-\alpha)[\lambda(k-1)+1] \\
& \cdot\left\{[\lambda(k-1)+1]^{r-1}(k-\alpha)^{r}-m(p-\alpha)^{r}[\lambda(p-1)+1]^{r-1}\right\}^{2}>0, \\
C(k)= & p(k-\alpha)[\lambda(k-1)+1] m^{2}(p-\alpha)^{2 r}[\lambda(p-1)+1]^{2(r-1)} \\
& +p m(p-\alpha)^{r}[\lambda(p-1)+1]^{r-1}(k-\alpha)^{r}[\lambda(k-1)+1]^{r-1} \\
& \cdot\{\lambda(k-p)(k-\alpha)(r-1)+[\lambda(k-1)+1][(k-p)(r-1)-(p-\alpha)]\} .
\end{aligned}
$$


It is clearly $C(k) \geq C(n+p)$, using (2.8), we obtain

$$
\begin{aligned}
& C(k) \geq C(n+p) \geq m^{2}(p-\alpha)^{2 r}[\lambda(p-1)+1]^{2(r-1)} \\
& \cdot\{[\lambda(n+p-1)+1][p(p-\alpha)+n(r-1)+p(n-1)]+\lambda n(n+p-\alpha)(r-1)\} \geq 0 .
\end{aligned}
$$

We see that $g^{\prime}(k)=\frac{C(k)}{D(k)} \geq 0$, this implies $g(k)$ is increasing in $\mathrm{k}$, which gives that

$$
\begin{aligned}
g(k) & \geq g(n+p) \\
& =p-\frac{m n(p-\alpha)^{r}[\lambda(p-1)+1]^{r-1}}{(n+p-\alpha)^{r}[\lambda(n+p-1)+1]^{r-1}-m(p-\alpha)^{r}[\lambda(p-1)+1]^{r-1}} .
\end{aligned}
$$

From $p(n+p-\alpha)^{r}[\lambda(n+p-1)+1]^{r-1} \geq m(n+p)(p-\alpha)^{r}[\lambda(p-1)+1]^{r-1}$, we can see that $0 \leq \gamma_{m}<p$, which complete the proof of Theorem 2 .

A special case of Theorem 2 when $\lambda=0$ and $\lambda=1$ yield

Corollary 3. (See [2, Theorem 3].) If the functions $f_{j}(z)=z^{p}-$ $\sum_{k=n+p}^{\infty} a_{k, j} z^{k} \in T_{n}\left(p, \alpha_{j}\right), j=1,2, \ldots, m$, define $F_{m}(z)=z^{p}-\sum_{k=n+p}^{\infty}\left(\sum_{j=1}^{m} a_{k, j}^{r}\right) z^{k}$ $(z \in \triangle ; n, p \in N ; r>1)$, then when

$$
p(n+p-\alpha)^{r} \geq m(n+p)(p-\alpha)^{r},
$$

we have $F_{m}(z) \in T_{n}\left(p, \beta_{m}\right)$, where

$$
\beta_{m}=p-\frac{m n(p-\alpha)^{r}}{(n+p-\alpha)^{r}-m(p-\alpha)^{r}}, \alpha=\min _{1 \leq j \leq m}\left\{\alpha_{j}\right\} .
$$

The result is sharp for the functions $f(z)$ given by

$$
f(z)=z^{p}-\frac{p-\alpha_{j}}{n+p-\alpha_{j}} z^{n+p}, j=1,2, \ldots, m ; n, p \in N .
$$

Corollary 4. (See [2, Theorem 4].) If the functions $f_{j}(z)=z^{p}-$ $\sum_{k=n+p}^{\infty} a_{k, j} z^{k} \in C_{n}\left(p, \alpha_{j}\right), j=1,2, \ldots, m$, define $F_{m}(z)=z^{p}-\sum_{k=n+p}^{\infty}\left(\sum_{j=1}^{m} a_{k, j}^{r}\right) z^{k}$ $(z \in \triangle ; n, p \in n ; r>1)$, then when

$$
(n+p-\alpha)^{r}(n+p)^{r-2} \geq m(p-\alpha)^{r} p^{r-2},
$$

we have $F_{m}(z) \in C_{n}\left(p, \xi_{m}\right)$, where

$$
\xi_{m}=p-\frac{m n(p-\alpha)^{r} p^{r-1}}{(n+p-\alpha)^{r}(n+p)^{r-1}-m(p-\alpha)^{r} p^{r-1}}, \quad \alpha=\min _{1 \leq j \leq m}\left\{\alpha_{j}\right\} .
$$

The result is sharp for the functions $f(z)$ given by

$$
f(z)=z^{p}-\frac{p\left(p-\alpha_{j}\right)}{(n+p)\left(n+p-\alpha_{j}\right)} z^{n+p}, \quad j=1,2, \ldots, m ; n, p \in N .
$$


Remark 2. (i). Putting $r=2$ and $\alpha_{j}=\alpha(j=1,2, \ldots, m)$ in Corollary 3 and Corollary 4, respectively, we obtain Owa [1, Theorem 5] and Owa [1, Theorem 6].

(ii). Taking $r=2, \alpha_{j}=\alpha(j=1,2, \ldots, m)$ and $n=p=1$ in Corollary 3 and Corollary 4, respectively, we obtain Owa [1, Corollary 9] and Owa [1, Corollary 10].

(iii). Taking $r=2, \alpha_{j}=\alpha(j=1,2, \ldots, m)$ and $p=1$ in Corollary 3 and Corollary 4, respectively, we obtain Domokos [4, Corollary 2] and Domokos [4, Corollary 3].

\section{The extreme points of $\phi_{c}^{*}(n, p, \lambda, \alpha)$}

Let $K$ be a subset of a vector space X, the extreme points of $K$ can be expressed as follows: $x_{o} \in E(K)$ if and only if the condition $x, y, x_{0} \in K$, $0<t<1$ and $t x+(1-t) y=x_{0}$ can make sure $x=y=x_{0}$, where $E(K)$ denote the set of all extreme points of $K$.

LEMMA 2. If the function

$$
f(z)=z^{p}-\frac{c(p-\alpha)[\lambda(p-1)+1]}{(n+p-\alpha)[\lambda(n+p-1)+1]} z^{n+p}-\sum_{k=n+p+1}^{\infty} a_{k} z^{k} \in S(n, p),
$$

then $f(z)$ is in the class $\phi_{c}^{*}(n, p, \lambda, \alpha)$ if and only if

$$
\begin{aligned}
& \sum_{k=n+p+1}^{\infty}(k-\alpha)[\lambda(k-1)+1] a_{k} \leq(1-c)(p-\alpha)[\lambda(p-1)+1] \\
& \quad(0 \leq c \leq 1) .
\end{aligned}
$$

Proof. Using Lemma 1, we can obtain the result of Lemma 2 easily.

Lemma 3. (See [8]). Suppose $X$ is a topological vector space. If $K$ is a nonempty compact subset in $X$, then $K \subset \overline{c o}(E(K))$. In particular, if $K$ is a nonempty compact convex set in $X$, then $K$ is the closed convex hull of the set of its extreme points.

LEMMA 4. Let

$$
f_{n+p}(z)=z^{p}-\frac{c(p-\alpha)[\lambda(p-1)+1]}{(n+p-\alpha)[\lambda(n+p-1)+1]} z^{n+p}
$$

and

$$
f_{k}(z)=z^{p}-\frac{c(p-\alpha)[\lambda(p-1)+1]}{(n+p-\alpha)[\lambda(n+p-1)+1]} z^{n+p}-\frac{(1-c)(p-\alpha)[\lambda(p-1)+1]}{(k-\alpha)[\lambda(k-1)+1]} z^{k}
$$

$(k \geq n+p+1)$, then $f(z) \in \phi_{c}^{*}(n, p, \lambda, \alpha)$ if and only if $f(z)=\sum_{k=n+p}^{\infty} \lambda_{k} f_{k}(z)$, where $\lambda_{k} \geqslant 0$ and $\sum_{k=n+p}^{\infty} \lambda_{k}=1$. 
Proof. Firstly, if $f(z)=\sum_{k=n+p}^{\infty} \lambda_{k} f_{k}(z)$, then we have

$$
\begin{aligned}
f(z)= & z^{p}-\frac{c(p-\alpha)[\lambda(p-1)+1]}{(n+p-\alpha)[\lambda(n+p-1)+1]} z^{n+p} \\
& -\sum_{k=n+p+1}^{\infty} \frac{(1-c)(p-\alpha)[\lambda(p-1)+1]}{(k-\alpha)[\lambda(k-1)+1]} \lambda_{k} z^{k}
\end{aligned}
$$

So

$$
\begin{aligned}
& \sum_{k=n+p+1}^{\infty}(k-\alpha)[\lambda(k-1)+1] \frac{(1-c)(p-\alpha)[\lambda(p-1)+1]}{k(k-\alpha)[\lambda(k-1)+1]} \lambda_{k} \\
& \quad=(1-c)(p-\alpha)[\lambda(p-1)+1] \sum_{k=n+p+1}^{\infty} \lambda_{k} \leq(1-c)(p-\alpha)[\lambda(p-1)+1] .
\end{aligned}
$$

from Lemma 2, we can know $f(z) \in \phi_{c}^{*}(n, p, \lambda, \alpha)$.

Conversely, suppose $f(z) \in \phi_{c}^{*}(n, p, \lambda, \alpha)$, then from Lemma 1 , it is easy to know that

$$
a_{k} \leq \frac{(1-c)(p-\alpha)[\lambda(p-1)+1]}{(k-\alpha)[\lambda(k-1)+1]} \quad(k \geqslant n+p+1) .
$$

Setting

$$
\begin{aligned}
\lambda_{k} & =\frac{(k-\alpha)[\lambda(k-1)+1]}{(1-c)(p-\alpha)[\lambda(p-1)+1]} a_{k} \quad(k \geqslant n+p+1), \\
\lambda_{n+p} & =1-\sum_{k=n+p+1}^{\infty} \lambda_{k},
\end{aligned}
$$

then $f(z)=\sum_{k=n+p}^{\infty} \lambda_{k} f_{k}(z)$.

THEOREM 3. The extreme points of the class $\phi_{c}^{*}(n, p, \lambda, \alpha)$ are given by $f_{k}(z)$ that are defined in Lemma 4.

Proof. Letting

$$
\begin{aligned}
V=\left\{f_{k}(z): f_{n+p}(z)\right. & =z^{p}-\frac{c(p-\alpha)[\lambda(p-1)+1]}{(n+p-\alpha)[\lambda(n+p-1)+1]} z^{n+p}, \\
f_{k}(z)= & z^{p}-\frac{c(p-\alpha)[\lambda(p-1)+1]}{(n+p-\alpha)[\lambda(n+p-1)+1]} z^{n+p} \\
& \left.-\frac{(1-c)(p-\alpha)[\lambda(p-1)+1]}{(k-\alpha)[\lambda(k-1)+1]} z^{k} \quad(k \geq n+p+1)\right\} .
\end{aligned}
$$


Suppose

$$
\begin{array}{r}
z^{p}-\frac{c(p-\alpha)[\lambda(p-1)+1]}{(n+p-\alpha)[\lambda(n+p-1)+1]} z^{n+p}-\frac{(1-c)(p-\alpha)[\lambda(p-1)+1]}{(k-\alpha)[\lambda(k-1)+1]} z^{k} \\
=t g_{1}(z)+(1-t) g_{2}(z),
\end{array}
$$

where $0<t<1, g_{i}(z)=z^{p}-\frac{c(p-\alpha)[\lambda(p-1)+1]}{\left(n+p_{\alpha}\right)[\lambda(n+p-1)+1]} z^{n+p}-\sum_{k=n+p+1}^{\infty} a_{k, i} z^{k}$ and $g_{i}(z) \in \phi_{c}^{*}(n, p, \lambda, \alpha)(i=1,2)$, then we have

$$
\frac{(1-c)(p-\alpha)[\lambda(p-1)+1]}{(k-\alpha)[\lambda(k-1)+1]}=t a_{k, 1}+(1-t) a_{k, 2} .
$$

Since $g_{i}(z) \in \phi_{c}^{*}(n, p, \lambda, \alpha),(3.2)$ gives

$$
a_{k, i} \leq \frac{(1-c)(p-\alpha)[\lambda(p-1)+1]}{(k-\alpha)[\lambda(k-1)+1]} \quad(i=1,2) .
$$

This implies that $a_{k, 1}=a_{k, 2}=\frac{(1-c)(p-\alpha)[\lambda(p-1)+1]}{(k-\alpha)[\lambda(k-1)+1]}$. Moreover, we can note that $a_{n, 1}=a_{n, 2}=0$ when $n \neq k$ so $g_{1}(z)=g_{2}(z)$, this gives us

$$
\begin{aligned}
f_{k}(z)= & z^{p}-\frac{c(p-\alpha)[\lambda(p-1)+1]}{(n+p-\alpha)[\lambda(n+p-1)+1]} z^{n+p} \\
& -\frac{(1-c)(p-\alpha)[\lambda(p-1)+1]}{(k-\alpha)[\lambda(k-1)+1]} z^{k} \in E \phi_{c}^{*}(n, p, \lambda, \alpha) .
\end{aligned}
$$

Taking the same process, we can obtain:

$$
f_{n+p}(z)=z^{p}-\frac{c(p-\alpha)[\lambda(p-1)+1]}{(n+p-\alpha)[\lambda(n+p-1)+1]} z^{n+p} \in E \phi_{c}^{*}(n, p, \lambda, \alpha) .
$$

So $V \subset E \phi_{c}^{*}(n, p, \lambda, \alpha)$. From Lemma 4, we know $\phi_{c}^{*}(n, p, \lambda, \alpha)=H V$. Since $V$ is a compact set, using Lemma 3, it gives $E \phi_{c}^{*}(n, p, \lambda, \alpha)=E H V \subset V$. So $E \phi_{c}^{*}(n, p, \lambda, \alpha)=V$.

\section{References}

[1] S. Owa, The Quasi-Hadamard products of certain analytic functions, in: H. M. Srivastava, S. Owa (eds.), Current Topics in Analytic Function Theory, World Scientific Publishing Company, Singapore, New Jersey, London, Hong Kong, 1992, 234-252.

[2] H. E. Darwish, M. K. Aouf, Generalizations of modified-Hadamard products of p-valent functions with negative coefficients, Math. Comput. Modelling (2007), doi:10. 1016/j. mcm. 2007. 08. 016

[3] H. M. Srivastava, S. Owa, S. K. Chatterjea, A note on certain classes of starlike functions, Rend. Sem. Mat. Univ. Padova 77 (1987), 115-124.

[4] T. Domokos, On a subclass of certain starlike functions with negative coefficients, Studia Univ. Babeş-Bolyai Math. (1991), 29-36. 
[5] O. Altintaş, H. Irmak, H. M. Srivastava, Fractional calculus and certain starlike functions with negative coefficients, Comput. Math. App. 30(2) (1995), 9-16.

[6] O. Altintas, Ö. Özkan and H. M. Srivastava, Neighborhoods of a certain family of multivalent functions with negative coefficients, Comput. Math. App. 4(7) (2004), 16671672 .

[7] O. Alt1ntaş, On a subclass of certain starlike functions with negative coefficients, Math. Japonica 36(3) (1991), 1-7.

[8] W. Rudin, Functional Analysis, Second Edition, China Machine press, BeiJing, 2004.

THE COLLEGE OF ENGINEERING AND TECHNICAL

CHENGDOU UNIVERSITY OF TECHNOLOGY

LESHAN, SICHUAN, 614000, CHINA

E-mail: xlpwxf@163.com

Received January 4, 2009; revised version April 30, 2010. 